\section{Military Technical College}

Kobry El-Kobbah,

Cairo, Egypt

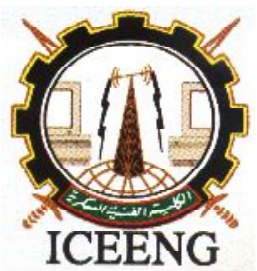

ICEENG $10^{\text {th }}$ International Conference

on Electrical Engineering

ICEENG 2016

\title{
A Real Time Control System Simulation Model Based on LabVIEW Graphical Programming Language
}

\author{
M.AHMED ${ }^{*}$, H. S. TAHA ${ }^{\dagger}$, A.SAAID ${ }^{\ddagger}$, M.TAREK $\ddagger$
}

\begin{abstract}
Embedded control system typically requires an architecture that is designed for reliability and deterministic performance. As a result, real time control system requires processes dedicated to system status monitoring, error handling and watchdog timers. This paper present a six degree-of-freedom (6-DOF) missile model implemented using structured graphical model building blocks. This model illustrates how a graphical representation of a missile model leads to a much clearer representation of the original mathematical model. The LabVIEW graphical programming software and collocate the Real Time/ (6-DOF) model software module are used to establish the missile behavior and nature which integrate the software and hardware equipment. LabVIEW includes tools for low-level system debugging and precise execution timing so that it can increase the flexibility and development of the missiles. Real time simulation is growing in its importance to the success of these systems.A validation of the developed model design is performed through software in loop simulation (SIL). This is to ensure the safety and economics, as the real flight test cannot easily be done.
\end{abstract}

Keywords:Embedded control system, simulation model, graphical programming, 6DOF, software in the loop (SIL)

\section{Introduction}

This paper presents a simulation for a complete nonlinear 6-DOF missile simulation model for a ballistic missile using implemented structured graphical blocks. The profile of the ballistic trajectory from launch to impact point can be divided into two phases, the active guided phase and the passive unguided phase.The model is a simulation for the active guided phase only, which lasts from the launching time to missile motor thrust burnout, because the guidance of the ballistic missile occurs entirely during this active phase. This model consists of mainly of six hierarchy graphical blocks. The model

*Graduate Student, Elec. Dept., MTC, Cairo, Egypt

${ }^{\dagger}$ Assistant Professor, Army Research \& Development Center, Cairo, Egypt

\# Professor, Elec. Dept., MTC, Cairo, Egypt

¥Assistant Professor, Elec. Dept., MTC, Cairo, Egypt 
contains simple simulated blocks for the real ballistic missile that can be replaced simply and quickly by other blocks using drag and drop features for the development and enhancement purpose.

For investigating the performance of the system and keeping with the requirements for a continued development, the model is structured as a series of modules. These modules can be individually developed for instance; there are airframe structure module, thrust variation module, weight data module, variation of missile mass center module, variation of mass center of oxidizer and fuel tanks, gravity module, earth module, variation of inertial moment module, and atmospheric data module.LabVIEW ${ }^{\mathrm{TM}}$, a modeling and implementation environmentwill be introduced, which is more useful for those who would like to take advantage of the benefits from implementations in reconfigurable hardware. Graphical programming and design can improve development experience and productivity by making design intuitive with library block constructs and offering a powerful integrated modeling, analysis, and deployment environment[1].This paper is organized into 5 sections. Section 2 illustrates the real time flight control system. Section 3introducesthe LabVIEW graphical programming environment. Section 4 presents the proposed mathematical 6-DOF simulation model,the function of each graphical block for the model and the benefits of graphical programming. Simulation results and analysis are discussed in section 5. Paper terminated with the conclusion in section 6.

\section{Real time embedded flight control system}

In this section a surveying for the real time operating system (RTOS) will be presented such that the target machine will include a VxWorks RTOS.A brief of the flight control system types and its main components for missile systemis illustrated. The real time flight control system is the most significant issue for the ballistic missile in the guided active phase and it will be the point of interest in this research.

\subsection{Real time operating system (RTOS)}

A real time operating system is the ability of the operating system to provide a required level of service in a bounded response time [6]. RTOS are classified into two categories [7]: Hard real time: negligible degree of tolerance to missed deadlines and if the time is more than system sampling time, it can result in system failure. Soft real time: The time can be lost from time to time, but the system does not fail and the system's quality is acceptable. The features of RTOS are multithreading, short latencies, task priority and inter-tasks communication [6, 7]. Limitations of RTOS are [6]: It can be costly, RTOS are generally complicated and can consume a non-trivial amount of processor cycles, and RTOS doesn't support multitasking with absence of round-robin scheduling which means the sequence of task from one to other in circular fashion like task 1, 2, 3, 4, 1, 2, 3, 4... [7]. Examples for RTOS: VxWorks, Windows CE, QNX Neutrino, RTAI, LynxOS, Micrium $\mu \mathrm{C} / \mathrm{OS}-\mathrm{II}$, Jbed and Symbian [6, 7].

\subsection{Flight control system simulation model types and components}

In this section, theflight control system simulation model types and its components will be introduced. The main four basic elements of the flight control system are shown in the 
gray box of Fig.1 []. The inertia measuring unit (IMU) senses the inertial motion of the missile. Its outputs and the inputs from the guidance law are combined in the autopilot to form a command input to the control effector, such as the commanded deflection angle to an aerodynamic control surface. The actuator turns the autopilot command into the physical motion of the control effector, which in turn influences the airframe dynamics to track the guidance command.

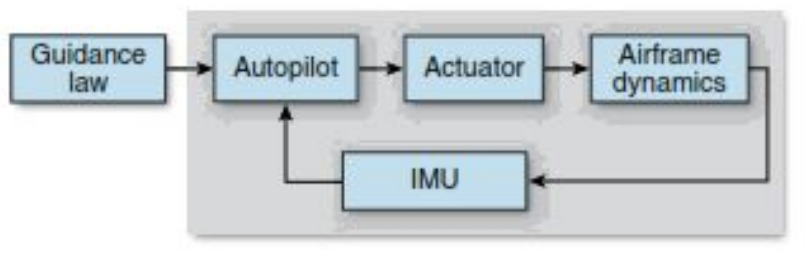

Fig. 1. Components of flight control system

The specific type of flight control system that is implemented on a particular missile depends on several factors, including the overall system mission and requirements, packaging constraints, and cost. There are three major types of flight control system[5] :

1. Acceleration Control System: deflection of an aerodynamic control surface, such as a tail fin, is the control input, and pitch angular rate and acceleration are measured by the IMU for feedback to the autopilot. The control deflection produces a small aerodynamic force on the tail fin but a large moment on the airframe because of its lever arm from the center of mass. The induced moment rotates the missile to produce the angle of attach(AOA), which in turn produces aerodynamic lift to accelerate the airframe.

2. Attitude Control System: another type of autopilot that can be used to control the attitude of the missile. In this case, the control effector is the thrust-deflection angle that is actuated by either a nozzle or jet tabs. It is used in this work.

3. Flight-Path Angle Control System: an autopilot that can be used to track flightpath angle commands using thrust-vector control. This type of system assumes that aerodynamic forces are small and hence applies for exoatmospheric flight or for endoatmospheric flight when the missile speed is low.

The main components of the control system can be classified by the following modules:

1. Airframe Dynamics: the objective of the flight control system is to force the missile dynamics to track the input command. The dynamics of the airframe are governed by fundamental equations of motion, with their specific characteristics determined by the missile aerodynamic response, propulsion, and mass properties.

2. Actuator: The missile actuator converts the desired control command developed by the autopilot into physical motion[5], such as rotation of a tail fin.

3. Autopilot: The autopilot is a set of equations that takes as inputs the guidance commands and the feedback measurements from the IMU and computes the control command as the output[5].

4. Inertial Measurement Unit: The IMU measures the missile dynamics for feedback to the autopilot. In most flight control applications, the IMU is composed of 
[5] accelerometers and gyroscopes to measure three components of the missile translational acceleration and three components of missile angular velocity.

\section{The LabVIEW Graphical programming environment}

LabVIEW TM (Laboratory Virtual Instrumentation Engineering Workbench) was introduced as a scientific software system by National Instruments Corporation (NI) around 1986. It incorporates a visual/graphical programming language called G, which is based on a structured homogeneous dataflow model of computation. $G$ is a strongly typed language [1]. It supports modular programming and hierarchical composition is accomplished in the form of a Virtual Instrument (VI). The LabVIEW Simulation Module provides a means of representing dataflow logic in control block diagram form typical in the design of control systems, and includes numerical ordinary differential equation solvers for simulation or real time implementation [3].LabVIEW Real Time extends the LabVIEW graphical development environment to deliver deterministic, hard real time performance. The LabVIEW RT architecture consists of the following three components [4]: LabVIEW software, the Real Time Development System and the Real Time Engine as shown in Fig 2.

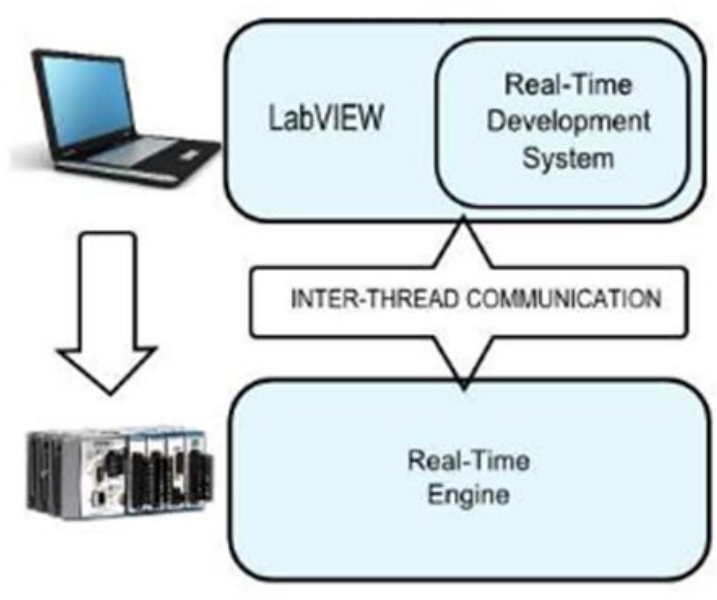

Fig. 2. Components of LabVIEW Real-Time Architecture

CompactRIO is an example of Real Time engine which bases on new reconfigurable I/O (RIO) technology and is combines the processing power and flexibility of a fieldprogrammable gate array (FPGA) with the reliability of a real-time processor. Include three components[4] as shown in Fig. 3:

1. Processor to execute LabVIEW Real Time applications for reliable real time operating system (VxWorksRTOS).

2. Reconfigurable embedded chassis with programmable FPGA core that can be accessed and configured using LabVIEW graphical development software;

3. Hot-swappable industrial I/O modules with built-in signal conditioning for direct connection to a variety of sensors and actuators. 


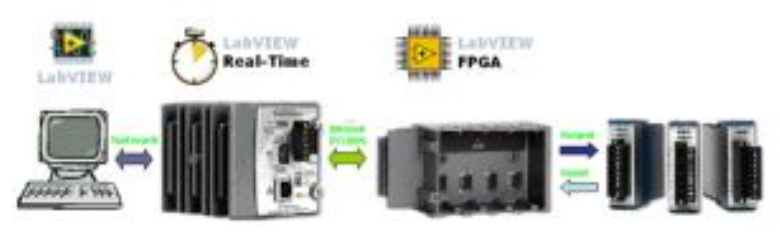

Fig.3. Components of CompactRIO

\section{Proposed 6-DOF mathematical model simulation for ballistic missile}

The 6-DOF simulation coded from the proposed missile system specifications can be used to verify the predicted performance of the system once the full aerodynamic characteristics, functional algorithms, and expected noise sources are included. A 6-DOF simulation can be used to design initial flight tests to exercise various missile subsystems at particular operating conditions. Similarly, after flight testing of the system has begun the 6-DOF simulation parameters can be validated against the measured telemetry. The simulation can be used to assess the risks associated with modifications to the missile or to assess its performance against a new threat. For an operational system, a simulation identifies and illuminates key sensitivities in the existing hardware. The effect on system responsiveness and lethality of potential hardware or software changes can be characterized. Understanding this system behavior allows the engineer to revise the missile specifications, if necessary ,for future deployments. Finally, it can be used to generate operational guidelines for deployment and firingprotocols. Fig. 4 illustrates the representation of the missile developed 6-DOF orientation in body coordinates $(x, y$, and z).Moreover, Fig. 4 illustrates that, the symbols $\vartheta, \psi, \varphi$ are the pitch, yaw, roll angles of the missile respectively. Moreover, the symbols $\dot{\hat{\theta}}, \dot{\psi}, \dot{\varphi}$ are the pitch, yaw, roll angles rates of the missilerespectively. $\alpha$ is the angle of attack, $\beta$ is the slip angle, $\Psi$ is the trajectory turning angle and $\theta$ is the missile flight path angle, $V_{m}$ is the velocity vector. The illustration of the most important parameters for the missile simulation model will be introduced in the experimental results section.

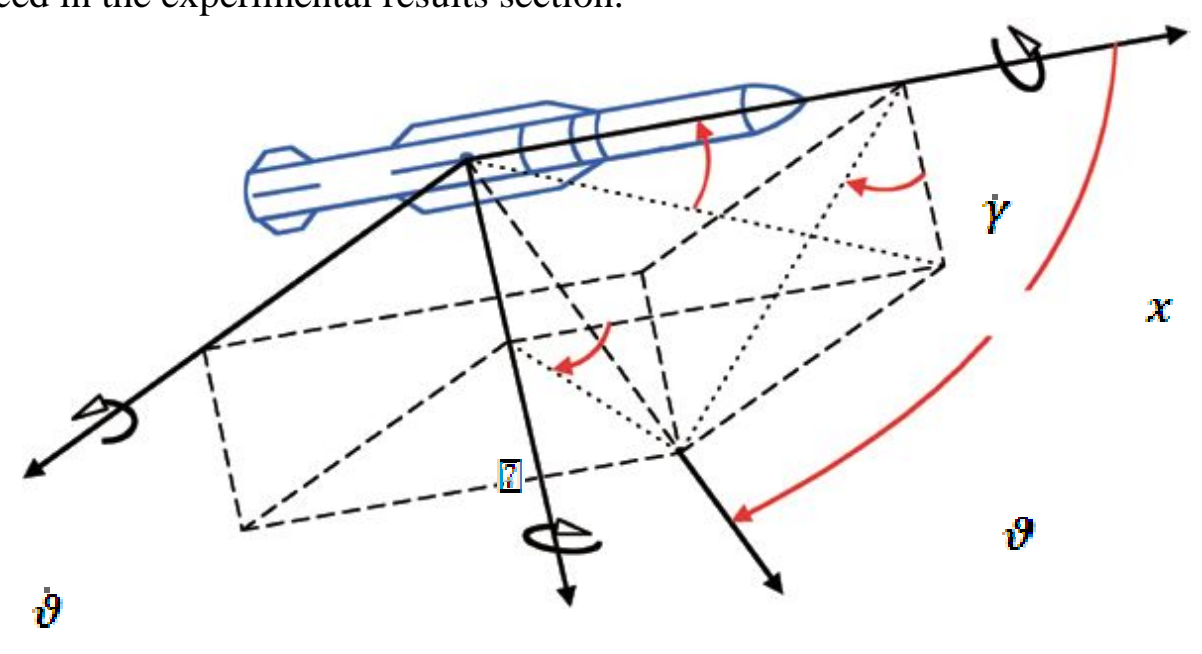

$Z$

Fig. 4. The missile 6-DOF cowdinate system $(x, y$, and $z)$

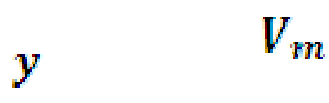


The 6-DOF model is developed using the graphical programming language LabVIEW that provides a very important abstraction mechanism that helps to increase the readability and understandability of a program. The development and implementation of the model are performed in the host and real time target machines. The diagram of a VI can optionally have an icon that suggests its functionality. The icon associates a number of the interactive control inputs with wiring connections on the icon so that data can be passed to and from the VI diagram[4]. This allows easy re-use of LabVIEW modules and supports the hierarchical composition of structured dataflow diagrams. Complex diagrams can be made more manageable by breaking them into modular pieces. It seems that this approach seems very natural and intuitive to most users. The mathematical simulation model is simulated such that modularity, readability and structure programming concepts are achieved to facilitate system development and enhancement. The general structure block diagram is illustrated as shown in Fig. 5. Different block functions are explained with the important input and output parameters that have the most powerful effect on the model.

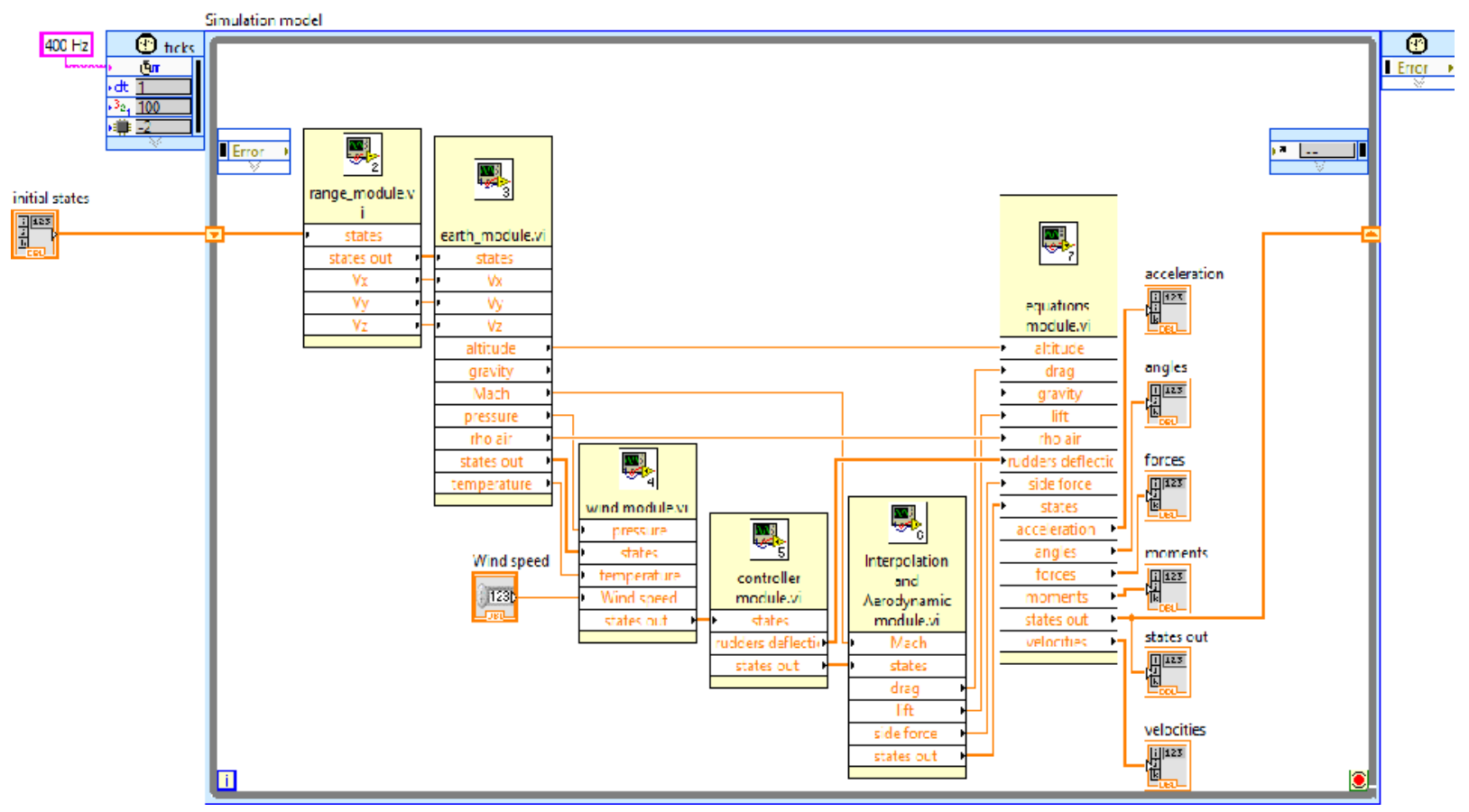

Fig.5. General block diagram for 6-DOF of the missile

The description for the main six developed function modules of the 6-DOF mathematical model can be presented as follows:

1. Range Controlmodule: The range control module is responsible for calculating the shutoff time of the missile engine based on themissile velocities. A certain range is settled by the user in the pre-launch phase.This module is responsible for calculating the requiredrange according to the three velocities of the missile.

2. Earth model module:All data about the earth and the effect of the earth rotation on missile parameters are included in the earth model.It entirely contains a graphical 
block responsible for calculating the Mach number and the pressure that affect the missile according to the altitude reached.The gravity of earth is also included in that model where the gravity across different height is calculated;Carioles effect is taken into consideration.

3. Wind module:Disturbance is an important factor that must be studied well, where it affect the angle of attack of the missile by having a data for the wind speed, atmospheric pressure and temperature, then calculating the effect of them on missile velocity and angle of attack.

4. Controller module:The actuator system is presented including the rudders and servo mechanical system, autopilot mathematical model and the generated trajectory profile are included for controlling the missile body. The output of the model is the fin commands that are sent to the Fins/Actuators Model.The main task of the proposed real time control system is the calculation of the proportional integral derivative (PID) as follows [9].

$$
\vec{\delta}(t)=K_{p} \vec{e}(t)+K_{I} \int_{0}^{t} \vec{e}(\tau) d \tau+K_{d} \frac{d}{d t} \vec{e}(t)
$$

Where:

$\overrightarrow{\mathrm{e}}(\mathrm{t})$ is the error vector between the req

$\boldsymbol{V}_{\boldsymbol{z} \text { velocity. }} \mathbf{K}_{\mathrm{p}}, \mathbf{K}_{\mathrm{I}}, \mathbf{K}_{\mathrm{dl}}$ are thegains, tuning purameters of the missile. $\vec{\delta}(\mathbf{t})$ is the output commands for the servo systemangle the rudder deflections.

5. Interpolation and Aerodynamic module:Mathematical interpolation is used for finding new points for a given range of non-continuous points, so new points are generated by this module given two successive Mach number and producing the drag, lift and side forces affecting the missile.

6. Equations module: All produced parameters from previous blocks are the input for the last block which contains all mathematical equations to produce forces needed to move rudders, moments affecting the body, angles and rates of the moving body.

\section{Simulationresults and performance analysis}

The resulting model is graphically organized in a way that lends itself to intuitive navigation, even by those who did not participate in its development. Moreover, one of the most power features in the LabVIEW is model analysis where LabVIEW provide very precise tool to measure the execution time in one microsecond precision. The real time control system development on the host machine and real time engine (CompactRIO) could be analyzed to measure the execution time for the effective functions in the model, so these results are available for enhancement purpose. Once the simulation is assembled, the plotting capability and the ease in which constants could be 
changed made it quick and easy to debug and fine-tune the model.A validation of the developed model design is performed through software in loop simulation (SIL) within the LabVIEW platform. This is to ensure the safety and economics, as the real flight test cannot easily be done. As a result ofcompletes simulation run, an illustration of the most important parameters for the missile simulation model will be introduced.The following simulated results represent the active guided phase of the ballistic trajectory only.

Angles of attack $\boldsymbol{\alpha}$ and slip angle $\boldsymbol{\beta}$ are represented as shown in Fig. 6.Fig.7represents the simulated Pitch, Yaw, Roll angles and angle es (IMU and Rate Gyro) withPitch profile (command) angle and its angle depicts angles and right column introduces the angle rates.Fig. 8demonstrates the three simulated velocities $\left(\boldsymbol{V}_{x}, \quad \boldsymbol{V}_{y} \text { and } \boldsymbol{V}_{z}\right)_{\text {Of themissiles in the body axis.Fig. } 9 \text { illustrates }}$ the commanded control rudder deflection angles $\left(\sigma_{1}, \sigma_{2}, \sigma_{3}\right.$ and $\left.\sigma_{4}\right)$ which are implemented in the PID controllermodule based on the simulatedangles and ratesin Fig. 7 in addition to $\boldsymbol{V}_{\boldsymbol{z}}$ in Fig. 8.c.

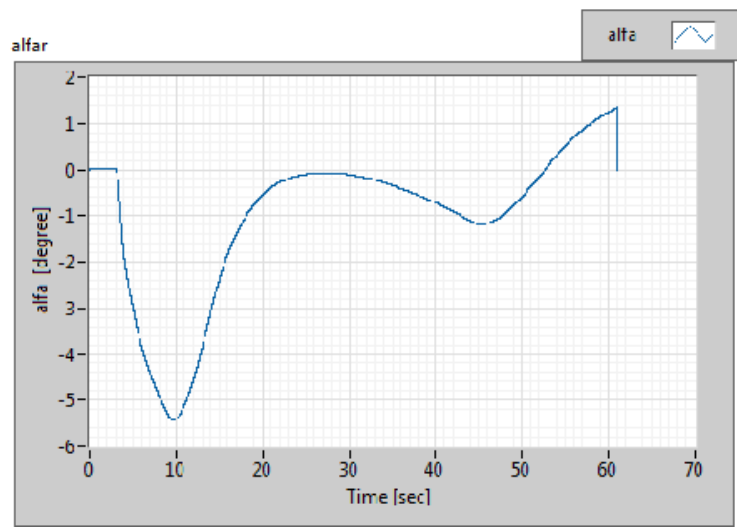

(a)

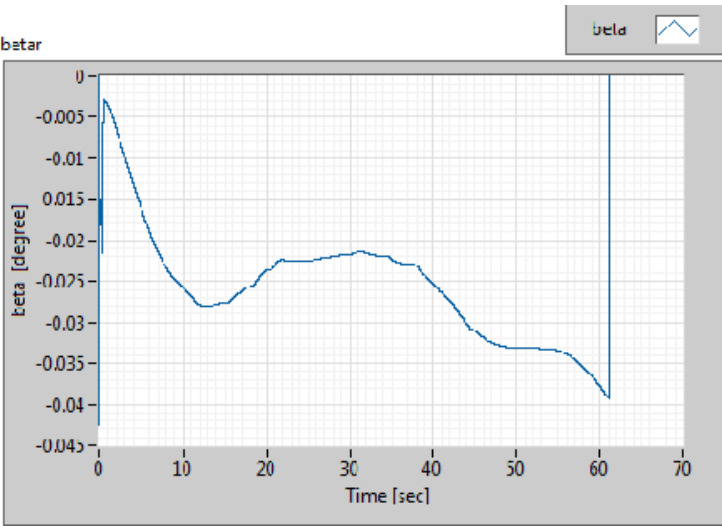

(b)

Fig. 6. Angles of attack $\alpha$ and slip angle $\beta$

\section{Conclusion}

In this research, the LabVIEW, graphical programming language provided an environment to develop the 6-DOF Missile model. A real time flight simulation was developed as a benchmark to demonstrate the value of graphical modeling. With its many point and click features, model development became easier and faster either by developing new model blocks or utilizing existing blocks from the LabVIEW modules. The resulting model is graphically organized in a way that lends itself to intuitive 
navigation, even by those who did not participate in its development. Once the simulation is assembled, the plotting capability and the ease in which constants could be changed made it quick and easy to debug and fine-tune the model. Individual deterministic analysis and visualization is easily completed in LabVIEW. Finally, softwareintheloop simulation was performed to analysis the controller quality. Thus, with the advantage of rapid prototyping using LabVIEW, it's efficient and convenient to design a real time simulation system with low cost of time and well expandability.

\section{References}

[1] G. Wang, T. N. Tran, and H. A. Andrade, "A graphical programming and design environment for FPGA-based hardware," in Field-Programmable Technology (FPT), 2010 International Conference on, 2010, pp. 337-340.

[2] P. A. Hawley and R. A. Blauwkamp, "Six-degree-of-freedom digital simulations for missile guidance, navigation, and control," Johns hopkins APL technical digest, vol. 29, pp. 71-84, 2010.

[3] A. Gancarczyk, "Real Time Measurement Systems Based On LabVIEW Graphical Environment," XI International PhD Workshop, 2009.

[4] R. Jamal and L. Wenzel, "The applicability of the visual programming language LabVIEW to large real-world applications," in Visual Languages, Proceedings., 11th IEEE International Symposium on, 1995, pp. 99-106.

[5] P. B. Jackson, "Overview of missile flight control systems," Johns hopkins APL technical digest, vol. 29, pp. 9-24, 2010.

[6] P. Hambarde, R. Varma, and S. Jha, "The Survey of Real Time Operating System: RTOS," in Electronic Systems, Signal Processing and Computing Technologies (ICESC), 2014 International Conference on, 2014, pp. 34-39.

[7] K. V. Prashanth, P. S. Akram, and T. A. Reddy, "Real time issues in embedded system design," in Signal Processing And Communication Engineering Systems (SPACES), 2015 International Conference on, 2015, pp. 167-171.

[8] S. C. Prodduturi, "A Six-degree-of-freedom Launch Vehicle Simulator for Range Safety Analysis," University of Florida, 2007.

[9] A. L. Greensite, Analysis and design of space vehicle flight control systems vol :11 .spartanBOOks New York, 1970. 


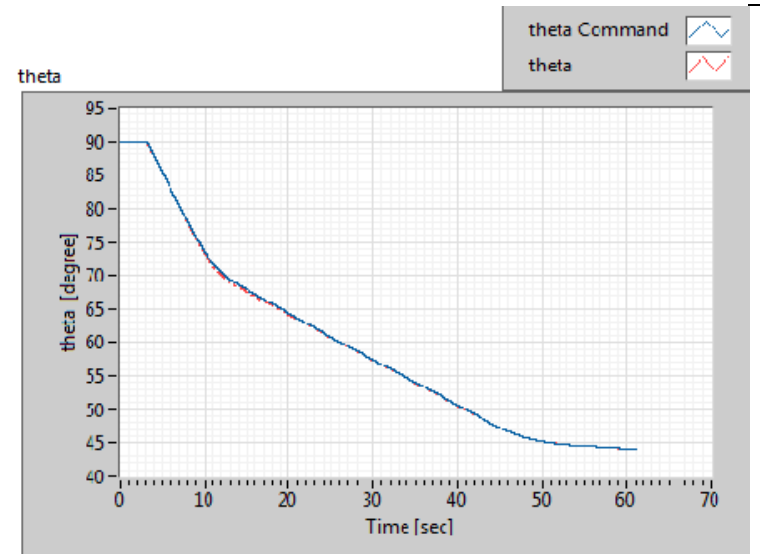

(a)

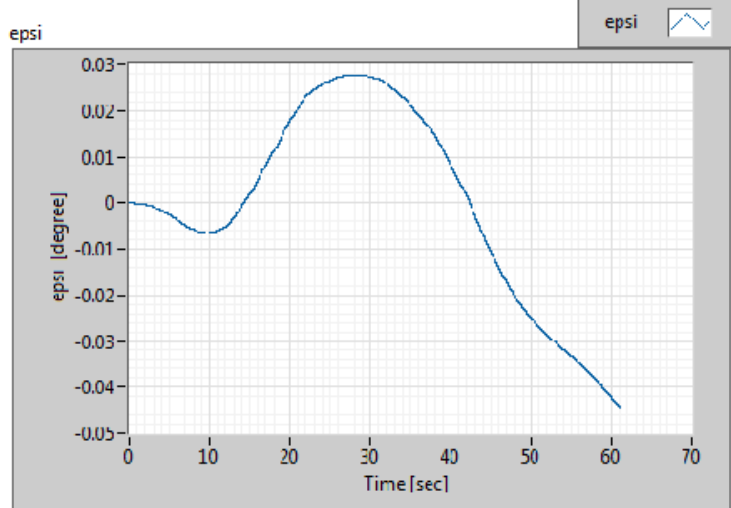

(c)

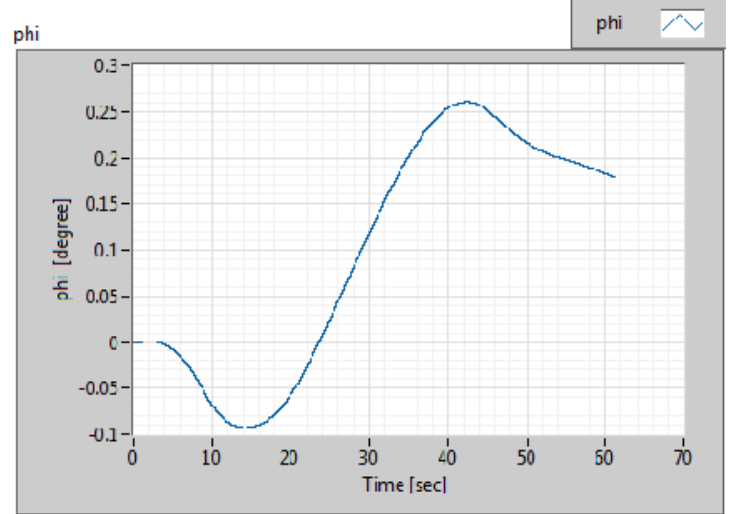

(e)

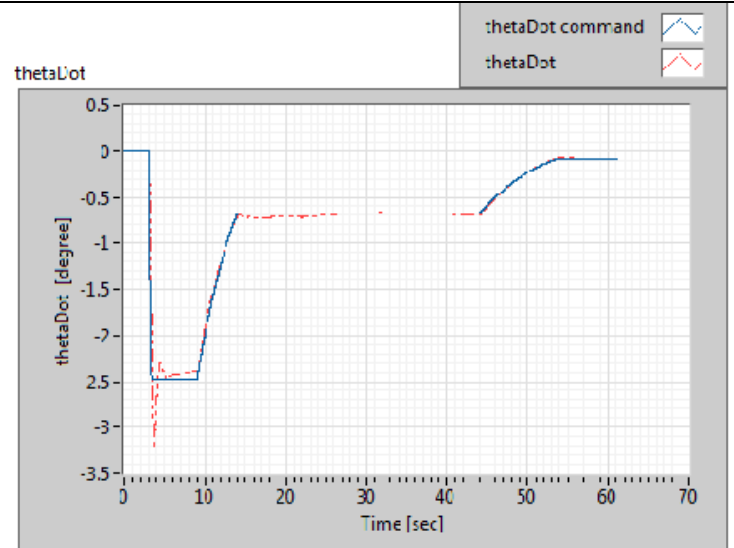

(b)

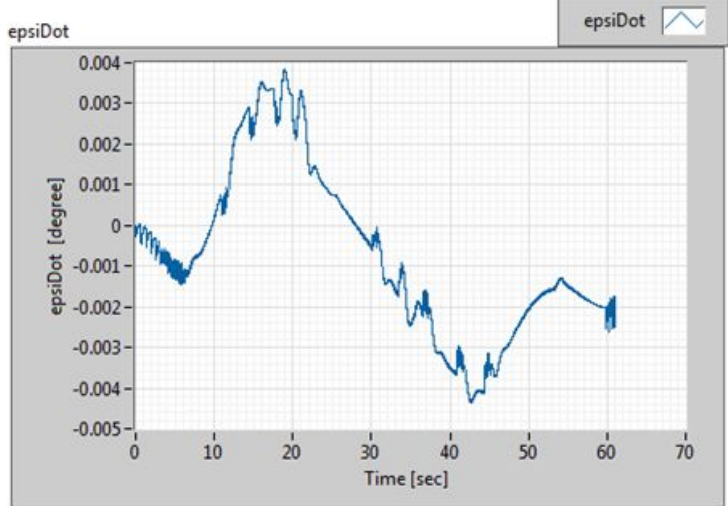

(d)

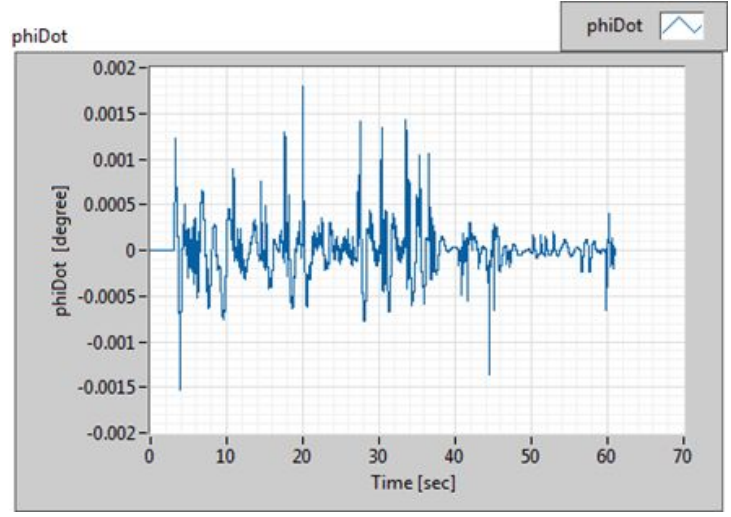

(f)

Fig.7. Pitch,Yaw, Roll angles and angle rates withPitch profile (Command) angle and itsangle rate: $\vartheta^{\vartheta}, \psi, \varphi, \dot{\vartheta}, \dot{\psi}, \dot{\varphi}, \vartheta_{p}$ and $\vartheta_{p}^{\prime}$ 


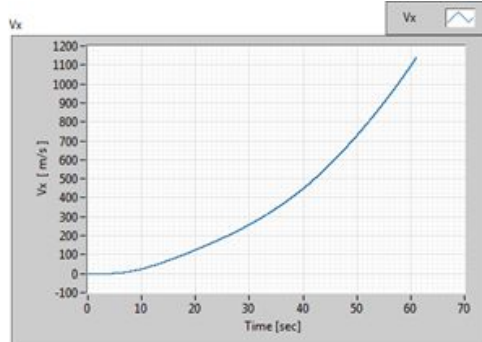

(a)

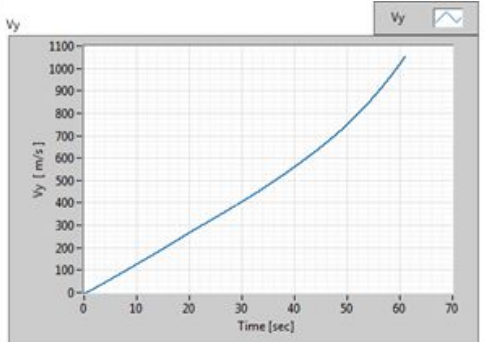

(b)

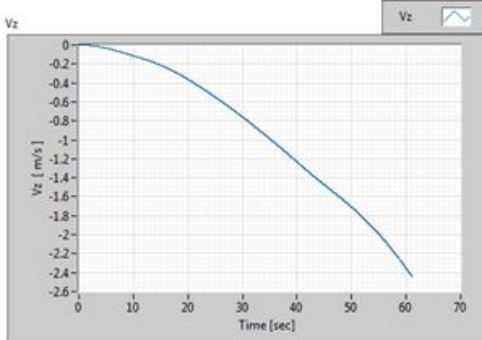

(c)

Fig. 8.The Three velocities in body axis $V_{x} V_{y} V_{z}$

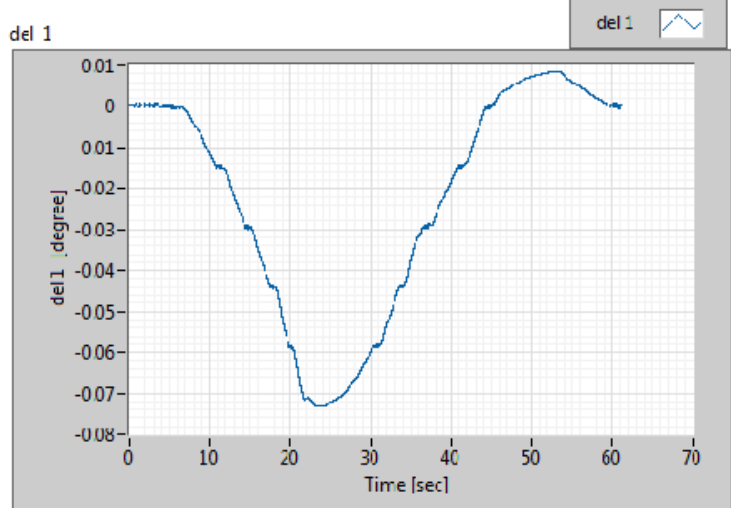

(a)

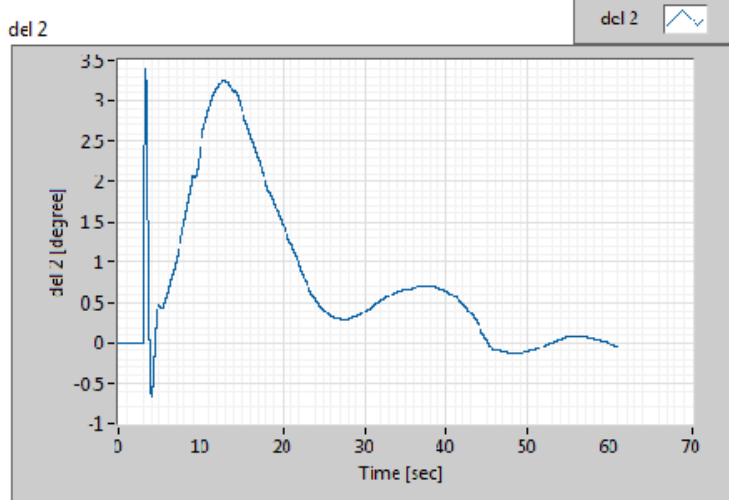

(c)

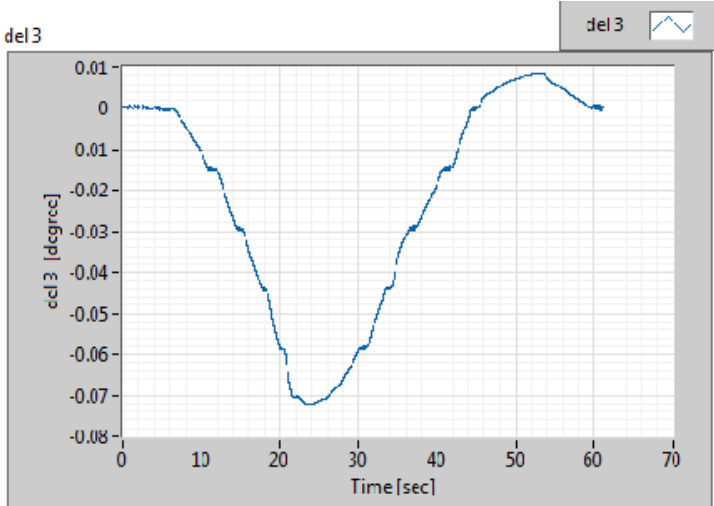

(b)

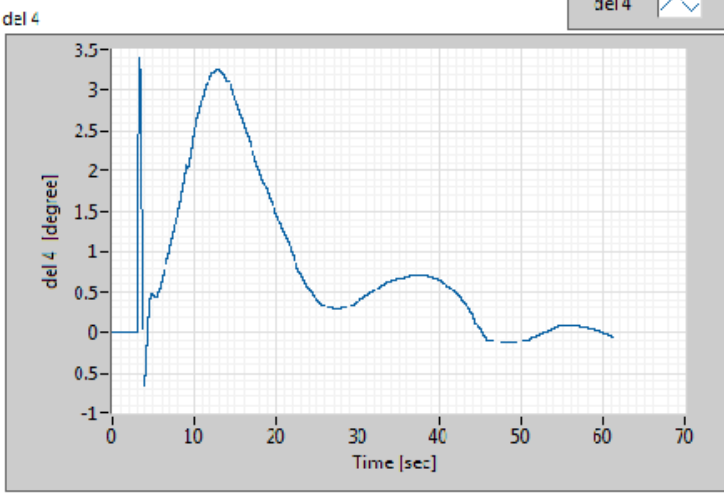

(d)

Fig.9. Rudder deflection $\left(\sigma_{1}, \sigma_{2}, \sigma_{3}\right.$ and $\left.\sigma_{4}\right)$ 